\title{
Intraoperative Respiratory Injury, CTCAE
}

National Cancer Institute

\section{Source}

National Cancer Institute. Intraoperative Respiratory Injury, CTCAE. NCI Thesaurus. Code C143611.

A finding of damage to the respiratory system during a surgical procedure. 